\title{
Qualidade de vida e nível cognitivo de pessoas idosas participantes de grupos de convivência
}

\section{Qdity of lifeandcogitiveled of edely mentbers of convivility graps}

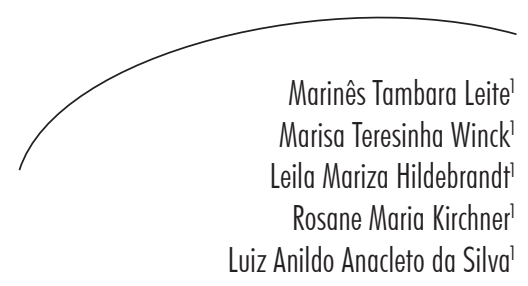

Resumo

Esta investigação teve o objetivo de avaliar a qualidade de vida e o nível cognitivo de idosos participantes de grupos de terceira idade de um município do Rio Grande do Sul, Brasil. Estudo com abordagem quantitativa, com 85 idosos. Para a coleta de dados foram utilizados o Mini-exame do Estado Mental (MEEM) e o Short-Form Health Survey-36 (SF 36). Os dados foram analisados seguindo a estatística descritiva. Os resultados mostram predomínio do sexo feminino idade entre 60 e 69 anos, viúvos, com quatro a sete anos de ensino formal e que recebem de um a três salários mínimos de renda. O resultado do MEEM indica que 23,6\% dos participantes apresentam declínio cognitivo. Os valores dos domínios avaliados pelo SF 36 mostram que a pior média está no quesito dor (30,47); no domínio aspectos sociais, o valor foi de 56,74; no de saúde mental atingiu 59,36, enquanto que o estado geral de saúde e de vitalidade apresentaram a mesma média, 58,29. Destacam-se com melhores médias os domínios nos aspectos emocionais $(69,82)$ e aspectos físicos $(73,82)$. Os resultados ampliam o conhecimento relativo à qualidade de vida de pessoas idosas participantes de atividades grupais e subsidiam a elaboração de estratégias no sentido da promoção do envelhecimento ativo.

\section{Abstract}

This investigation aimed to evaluate the quality of life and the cognitive level of elderly participants in conviviality groups in a city of Rio Grande do Sul state, Brazil. The cognitive approach was used in this study, which included 85 elderly. The Mini-exam of Mental State was used for the data collection and the Short-Form Health Survey-36. The data was analyzed according to the descriptive statistics. The results show a predominance

\footnotetext{
Departamento de Ciências da Saúde, Centro de Educação Superior Norte do RS. Universidade Federal de Santa Maria. Palmeira das Missões, RS, Brasil.

Apoio: Artigo desenvolvido a partir de pesquisa realizada no Departamento de Enfermagem do Centro de Educação Superior Norte da Universidade Federal de Santa Maria/UFSM, com apoio da FAPERGS/ ARD. A pesquisa contou, também, com bolsista de Iniciação Científica FIPE/UFSM, 2009.

Correspondência / Correspondence

Marinês Tambara Leite

Universidade Federal de Santa Maria/Centro de Educação Superior Norte do Rio Grande do Sul - UFSM/ CESNORS.

Rua Floriano Peixoto, 776 - Centro

98700-000, Ijuí, RS, Brasil

E-mail: tambaraleite@yahoo.com.br
}

Palavras-chave: Idoso.

Envelhecimento. Qualidade de Vida. Cognição. Rio Grande do Sul. 
of females, between the ages of 60 and 69, widows, the ones who attended from four to seven years of formal education and that receive from one to three minimum wages income. The results of MEEM indicates that $23.6 \%$ of the study participants have a cognitive decline. The values of the domains assessed by SF 36 showed that the worst average is presented in the pain item (30.47); in the social aspects domain the value was 56.74; the mental health item reached 59,36, while the general health state and vitality presented the same average, 58.29. The domain of emotional aspects stands out with the best averages (69.82) and physical aspects (73.82). The results amplify the knowledge about the quality of life of elderly people participating of group activities and subsidize the developing of strategies towards the promotion of active aging.
Key words: Aged. Aging. Quality of life. Cognition. Rio Grande do Sul.

\section{INTRODUÇÃO}

A definição de qualidade de vida (QV), especialmente na velhice, envolve um panorama multidimensional, complexo e apresenta aspectos objetivos e subjetivos. Quanto aos fatores objetivos, estes se constituem na ausência de enfermidades ou de perdas das capacidades funcionais, isto é, centrados nos aspectos biológicos e epidemiológicos. ${ }^{1}$ Os aspectos subjetivos são descritos como o entendimento que a pessoa possui de sua posição na vida, no cenário da cultura e no contexto de valores. Também diz respeito à qualidade nos relacionamentos, realização pessoal, oportunidades de lazer, além de contemplar os objetivos, expectativas, padrões e preocupações. ${ }^{1}$

Entende-se que o termo "QV" é um construto que abarca diversos significados, pois reflete conhecimentos, experiências e valores de indivíduos e populações em diferentes épocas, ambientes e histórias, sendo, portanto, uma construção social com influência de distintas culturas. $^{2}$ Os resultados de pesquisa realizada sobre QV na velhice ${ }^{3}$ apontam que 49\% dos entrevistados mencionam que qualidade de vida está relacionada aos bons vínculos instituídos com a família, vizinhos e amigos.

Quando se trata de indivíduos que se encontram na velhice, a QV está atrelada à manutenção da saúde no âmbito físico, social, psíquico e espiritual. Autonomia, independência, boa saúde física, exercício de papéis sociais, atividade e senso de pertencimento são atributos de boa QV dos idosos. ${ }^{4}$
Entender o que significa QV para idosos no Brasil torna-se relevante, pois dados do Censo Demográfico do Instituto Brasileiro de Geografia e Estatística ${ }^{5}$ demonstram que, no início da década, esta população era de 11,4 milhões de brasileiros. Atualmente, o número de idosos representa 14,5 milhões de brasileiros, e a previsão para o ano de 2025 é que excederá os 30 milhões de indivíduos.

São diversas as questões que acompanham o envelhecimento da população. Com o aumento na expectativa de vida, a manutenção da capacidade funcional do idoso se torna importante, uma vez que poderá haver a preservação de sua independência, sobretudo para realizar as atividades de vida diária. Esta condição pode estar diretamente relacionada à QV do indivíduo.

Em se tratando especificamente da manutenção da capacidade funcional, uma das alternativas para sua melhoria é a realização de atividade física, uma vez que existe uma relação benéfica entre exercício físico e a preservação da capacidade funcional, pois os mesmos agem melhorando a saúde do indivíduo que permanece ativo e dá a ele maior independência física. ${ }^{6}$

Também, ao envelhecer, pode ocorrer diminuição no nível cognitivo em pessoas acima de 60 anos de idade. Esta constatação é bastante frequente, uma vez que os idosos, em geral, se queixam de dificuldades com a memória e outras habilidades cognitivas, particularmente quando comparam o desempenho atual com o do passado. ${ }^{7}$ Nesse cenário, torna-se necessário 
discutir e avaliar o nível de cognição da população idosa, condição diretamente relacionada a QV e de saúde da população idosa. Os estudos nesta área discutem basicamente dois aspectos da área cognitiva: uma ligada às perdas biológicas naturais e a outra atrelada ao tipo de cultura e ao contexto em que o idoso se encontra inserido. ${ }^{8}$

Os grupos de convivência oferecem estímulo para a melhoria do nível cognitivo, que pode ser por meio da realização de atividades manuais, artesanais, culturais, artísticas e da efetivação de técnicas de animação grupal e da dança. Dessa forma, o grupo oferece à pessoa idosa um aumento importante no suporte social. ${ }^{9} \mathrm{Na}$ velhice, tanto os recursos como as ações devem ser focadas ao bem-estar e a QV dos indivíduos que envelhecem. ${ }^{2}$

As atividades de lazer e a convivência grupal contribuem para a manutenção do equilíbrio biopsicossocial do idoso, além de abrandar possíveis conflitos ambientais e pessoais. $\mathrm{O}$ bem-estar proporcionado pela participação do idoso em atividades grupais coopera para que ele vivencie trocas de experiências e propicia conscientização para a importância do autocuidado. $\mathrm{O}$ aumento da expectativa de vida e a QV de idosos estão associados à evolução da tecnologia e da medicina, além da vivência destes em grupos, que ultrapassa somente a realização de atividades físicas e de lazer propostas, uma vez que envolvem aspectos emocionais e comportamentais, dentre outros. ${ }^{10}$ Assim, os grupos de convivência proporcionam espaço de interação, inclusão social, manutenção da autonomia, do viver com dignidade e, consequentemente, ser e estar saudável.

Tem-se como hipótese, para este estudo, que os idosos, ao frequentarem grupos de convivência, passam a ter uma boa qualidade de vida e bom nível cognitivo. Torna-se relevante, assim, investigar e acompanhar como está a qualidade de vida e o nível cognitivo de pessoas idosas que frequentam espaços sociais - no caso, grupos de convivência -, usufruindo de seus direitos como cidadãos com autonomia e independência.

\section{OBJETIVO}

Avaliar a qualidade de vida (QV) de idosos e o nível cognitivo de idosos participantes de grupos de terceira idade existentes em um município do Rio Grande do Sul/Brasil.

\section{MÉTODO}

A população investigada foi composta por idosos que frequentam grupos de terceira idade no município de Palmeira das Missões - RS, Brasil. Neste município há quatro grupos de terceira idade, que se reúnem sistematicamente, com o objetivo de realizar atividades tais como: artesanato, dança, passeios, exercícios físicos e socialização. Comumente, os encontros são semanais, coordenado por um profissional vinculado à Secretaria Municipal de Ação Social, e ocorrem em local próximo à residência dos idosos, geralmente em um pavilhão de uma igreja. Participam em cada grupo de 20 a 30 indivíduos, de ambos os sexos. Ressalta-se que alguns participantes possuem menos de 60 anos de idade.

Como critério de inclusão dos sujeitos, estabeleceu-se que fariam parte da pesquisa: indivíduos com 60 anos ou mais de idade; estar frequentando um dos grupos de terceira idade do município local de estudo; ter cursado pelo menos um ano o ensino formal; aceitar fazer parte da investigação; e assinar o termo de consentimento livre e esclarecido. Ficaram excluídos do estudo os indivíduos que não preencheram os requisitos preconizados. Desse modo, os sujeitos foram todos os 85 idosos que participam dos grupos de convivência pesquisados que atendiam os critérios de inclusão.

A coleta dos dados ocorreu no período de agosto a dezembro de 2009, com o auxílio de um instrumento que contém questões relativas aos dados sociodemográficos; o Mini-Exame do Estado Mental (MEEM) e o SF 36 (Medical Outcomes Study 36 - Short-Form Health Survey). O MEEM fornece informações sobre parâmetros 
cognitivos, com questões agrupadas em sete categorias. Cada uma objetiva avaliar "função" cognitiva específica: orientação temporal (5 pontos), orientação espacial (5 pontos), registro de três palavras ( 3 pontos), atenção e cálculo ( 5 pontos), recordação das três palavras (3 pontos), linguagem (8 pontos) e capacidade construtiva visual (1 ponto). O escore do MEEM pode variar de zero ponto, o qual indica o maior grau de comprometimento cognitivo dos indivíduos, até 30 pontos, que corresponde à melhor capacidade cognitiva. ${ }^{11}$ Destaca-se que, para este estudo, considerou-se portador de declínio cognitivo o idoso que apresentasse escore abaixo de 24 no MEEM. ${ }^{12}$

Para avaliar os aspectos referentes à QV, utilizou-se o SF-36 (Medical Outcomes Study 36 - Item Short-Form Health Survey), traduzido e validado para a língua portuguesa. ${ }^{13,14} \mathrm{O}$ SF-36 foi escolhido por ser um instrumento autoadministrável, podendo, entretanto, ser aplicado no formato de entrevistas. O SF-36 é um instrumento genérico de avaliação do estado de saúde, de fácil administração e compreensão, desenvolvido para ser utilizado em grupos, independentemente de faixa etária, patologia, tratamento, etnia ou sexo.

O SF-36 é multidimensional, formado por 36 itens, divididos em oito escalas ou domínios: capacidade funcional (CF), aspectos físicos (AF), dor física (DF), estado geral de saúde (EGS), vitalidade (VT), aspectos sociais (AS), aspectos emocionais (AE) e saúde mental (SM), em que cada componente ou domínio é avaliado separadamente. $\mathrm{O}$ questionário pode ser dividido em dois componentes: o componente físico envolve a capacidade funcional, a dor, o estado geral de saúde e o aspecto físico. $\mathrm{O}$ componente mental abrange a saúde mental, o aspecto emocional e o social e a vitalidade, que é avaliada por 35 questões. Além disso, há mais uma questão comparativa entre a saúde atual e a do ano anterior. A finalidade das questões foi transformar medidas subjetivas em dados objetivos, que permitem ser analisadas de forma específica, global e reprodutível. ${ }^{14}$

$\mathrm{Na}$ avaliação dos dados, utilizou-se um escore próprio para cada questão, em que o escore baixo reflete a má percepção da saúde, a perda da função e a presença de dor, enquanto o escore alto reflete boa percepção da saúde, função preservada e ausência de dor. Para avaliar os resultados finais, deu-se um escore de zero a 100 , em que zero equivale ao pior estado e 100 ao melhor estado de saúde. ${ }^{13}$

Para a análise dos dados, foi utilizado o software estatístico SPSS 8.0 for Windows (SPSS, Chicago, IL) e a estatística descritiva, envolvendo tabelas, médias e desvio padrão. Os aspectos éticos da pesquisa com seres humanos foram observados e o projeto de investigação foi aprovado pelo Comitê de Ética na Pesquisa da UFSM, sob o número 23081.017883/2008-03.

\section{RESULTADOS}

A caracterização dos sujeitos do estudo evidencia que $79(92,9 \%)$ são do sexo feminino e seis $(7,1 \%)$ do sexo masculino. Há predominância de idosos com idade entre 60 e 69 anos $(61,2 \%)$, seguidos dos que têm entre 70 e 79 anos (31,8\%). Já em relação à situação conjugal, verificase que a maioria dos idosos é viúvo (54,1\%), acompanhados de casados $(28,2 \%)$ e divorciados ou solteiros $(17,7 \%)$.

Quanto à escolaridade formal, a maior parte dos idosos frequentou de quatro a sete anos $(52,9 \%)$, seguido de um a três anos $(28,2 \%)$ e de oito anos ou mais $(18,8 \%)$. No quesito renda mensal, são maioria os idosos que recebem de um a três salários mínimos (88,2\%). Residem acompanhados $62,4 \%$ dos idosos, enquanto que $37,6 \%$ moram sós, dados que podem ser visualizados na tabela 1 . 
Tabela 1 - Distribuição dos idosos segundo características sociais e econômicas. Palmeira das Missões, RS, 2011.

\begin{tabular}{|c|c|c|c|}
\hline \multirow[b]{2}{*}{ Variáveis } & \multirow[b]{2}{*}{ Categorias } & \multicolumn{2}{|c|}{ Total } \\
\hline & & $\mathrm{n}$ & $\%$ \\
\hline \multirow{2}{*}{ Gênero } & Feminino & 79 & 92,9 \\
\hline & Masculino & 6 & 7,1 \\
\hline \multirow{3}{*}{ Idade } & 60 a 69 anos & 52 & 61,2 \\
\hline & 70 a 79 anos & 27 & 31,8 \\
\hline & 80 anos ou mais & 6 & 7,1 \\
\hline Situação & Viúvo & 46 & 54,1 \\
\hline \multirow{3}{*}{ Conjugal } & Casado & 24 & 28,2 \\
\hline & Desquitado/Divorciado & 13 & 15,3 \\
\hline & Solteiro & 2 & 2,4 \\
\hline \multirow{3}{*}{$\begin{array}{l}\text { Anos de } \\
\text { escolaridade }\end{array}$} & 1 a 3 anos & 24 & 28,2 \\
\hline & 4 a 7 anos & 45 & 52,9 \\
\hline & 8 anos ou mais & 16 & 18,8 \\
\hline \multirow[t]{2}{*}{ Religião } & Católica & 80 & 94,1 \\
\hline & Evangélica & 5 & 5,9 \\
\hline \multirow[t]{4}{*}{ Renda } & Menos de $1 \mathrm{SM}$ & 3 & 3,5 \\
\hline & De 1 a 3 SM & 75 & 88,2 \\
\hline & De 3 a 5 SM & 5 & 5,9 \\
\hline & Mais de $3 \mathrm{SM}$ & 2 & 2,4 \\
\hline Arranjo & Acompanhado & 53 & 62,4 \\
\hline Familiar & Mora sozinho & 32 & 37,6 \\
\hline
\end{tabular}

SM: Salários mínimos

O resultado do MEEM indica que 20 $(23,5 \%)$ participantes do estudo apresentam declínio cognitivo, cuja média de pontuação foi de 25,99 pontos, numa variação de 16 a 30 . Se forem considerados os idosos com 80 anos ou mais de idade, estes são os que apresentam maior incidência de declínio cognitivo nesta população. A relação entre a variável faixa etária e o nível cognitivo está expressa na tabela 2.

Tabela 2 - Distribuição dos idosos segundo os componentes do SF36 com e sem declínio cognitivo. Palmeira das Missões, RS, 2011.

\begin{tabular}{cccc}
\hline Idade & $\begin{array}{c}\text { Com Declínio } \\
\text { Cognitivo n }(\%)\end{array}$ & $\begin{array}{c}\text { Sem Declínio } \\
\text { Cognitivo } n(\%)\end{array}$ & $\begin{array}{c}\text { Total } \\
\mathrm{n}(\%)\end{array}$ \\
\hline 60 a 69 & $9(10,9)$ & $43(50,6)$ & $52(61,2)$ \\
70 a 79 & $7(8,2)$ & $20(23,5)$ & $27(31,8)$ \\
Mais de 80 & $4(4,7)$ & $2(2,4)$ & $6(7,1)$ \\
Total & $20(23,5)$ & $65(76,5)$ & $85(100)$ \\
\hline
\end{tabular}


Quanto aos valores dos domínios avaliados pelo SF 36, identifica-se que a pior média apresenta-se no quesito dor $(30,47 \pm 19,27)$, enquanto que as facetas estado geral de saúde e vitalidade possuem a mesma média de 58,29, embora o desvio padrão seja diferenciado 15,46 e 13,92, respectivamente. O nível de integração dos idosos em atividades sociais, que caracteriza os aspectos sociais, apresentou média

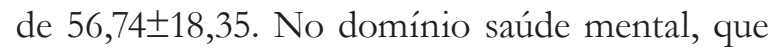
investiga ansiedade, depressão, alterações do comportamento e bem-estar psicológico, a média dos valores atingiu 59,36 $\pm 14,76$, o que pode ser observado na tabela 3 .

Tabela 3 - Valores dos domínios avaliados pelo SF 36. Palmeira das Missões, RS, 2011.

\begin{tabular}{lccccc}
\hline & $\mathrm{n}$ & li & ls & Média & $\begin{array}{c}\text { Desvio } \\
\text { padrão }\end{array}$ \\
\hline Capacidade Funcional & 85 & 20 & 100 & 69,88 & 20,10 \\
Aspecto Físico & 85 & 0 & 100 & 73,82 & 36,58 \\
Dor & 85 & 0 & 90 & 30,47 & 19,27 \\
Estado Geral de Saúde & 85 & 30 & 100 & 58,29 & 15,46 \\
Vitalidade & 85 & 20 & 95 & 58,29 & 13,92 \\
Aspectos Sociais & 85 & 25 & 100 & 56,74 & 18,35 \\
Aspectos Emocionais & 85 & 0 & 100 & 69,82 & 42,31 \\
Saúde Mental & 85 & 20 & 96 & 59,36 & 14,67 \\
\hline
\end{tabular}

$\mathrm{li}=$ Limite inferior; $1 \mathrm{~s}=$ Limite superior; $\mathrm{n}=$ total de entrevistados

$\mathrm{Na}$ tabela 4, apresentam-se os resultados do cruzamento do arranjo familiare a presença ou não de declínio cognitivo (declínio cognitivo quando escore abaixo de 24), ${ }^{12}$ para os diferentes domínios do SF 36. Destaca-se que, mesmo apresentando déficit cognitivo em todos os domínios, há idosos que residem sós no ambiente doméstico.
A tabela 5 mostra o cruzamento dos dados relativos à cognição - com e sem declínio - com a realização ou não de atividade física, associados aos domínios do SF 36. Observa-se que, entre os que apresentam déficit cognitivo, há uma maior realização de atividade física. 
Tabela 4 - Distribuição dos idosos segundo os componentes do SF36 com e sem declínio cognitivo e o arranjo familiar. Palmeira das Missões, RS, 2011.

\begin{tabular}{lcccc}
\hline \multirow{2}{*}{ Domínio } & $\begin{array}{c}\text { Arranjo } \\
\text { familiar }\end{array}$ & $\begin{array}{c}\text { Com Declínio } \\
\text { Cognitivo n(\%) }\end{array}$ & $\begin{array}{c}\text { Sem Declínio } \\
\text { Cognitivo n(\%) }\end{array}$ & $\begin{array}{c}\text { Total } \\
\mathrm{n}(\%)\end{array}$ \\
\hline \multirow{2}{*}{ Capacidade Funcional } & Acompanhado & $7(8,2)$ & $25(29,4)$ & $32(37,6)$ \\
& Mora sozinho & $9(10,6)$ & $44(51,8)$ & $53(62,4)$ \\
Aspecto Físico* & Acompanhado & $6(7,1)$ & $26(30,6)$ & $32(37,6)$ \\
& Mora sozinho & $20(23,5)$ & $33(38,8)$ & $53(62,4)$ \\
Dor & Acompanhado & $28(32,9)$ & $4(4,7)$ & $32(37,6)$ \\
\multirow{2}{*}{ Estado Geral de Saúde } & Mora sozinho & $49(57,6)$ & $4(4,7)$ & $53(62,4)$ \\
& Acompanhado & $11(12,9)$ & $21(24,7)$ & $32(37,6)$ \\
Vitalidade & Mora sozinho & $22(25,9)$ & $31(36,5)$ & $53(62,4)$ \\
\multirow{2}{*}{ Aspectos Sociais } & Acompanhado & $15(17,6)$ & $17(20,0)$ & $32(37,6)$ \\
& Mora sozinho & $17(20,0)$ & $36(42,4)$ & $53(62,4)$ \\
Aspectos Emocionais & Acompanhado & $25(29,4)$ & $7(8,2)$ & $32(37,6)$ \\
& Mora sozinho & $39(45,9)$ & $14(16,5)$ & $53(62,4)$ \\
Saúde Mental & Acompanhado & $10(11,8)$ & $22(25,9)$ & $32(37,6)$ \\
& Mora sozinho & $14(16,5)$ & $39(45,9)$ & $53(62,4)$ \\
& Acompanhado & $8(9,4)$ & $24(28,2)$ & $32(37,6)$ \\
& Mora sozinho & $10(11,8)$ & $43(50,6)$ & $53(62,4)$ \\
\hline
\end{tabular}

Tabela 5 - Distribuição dos idosos segundo os componentes do SF36 com e sem declínio cognitivo e prática de atividade física. Palmeira das Missões, RS, 2011.

\begin{tabular}{|c|c|c|c|c|}
\hline Domínio & $\begin{array}{l}\text { Atividade } \\
\text { física }\end{array}$ & $\begin{array}{l}\text { Com Declínio } \\
\text { Cognitivo } n(\%)\end{array}$ & $\begin{array}{l}\text { Sem Declínio } \\
\text { Cognitivo } n(\%)\end{array}$ & $\begin{array}{l}\text { Total } \\
\mathrm{n}(\%)\end{array}$ \\
\hline Capacidade Funcional & $\begin{array}{l}\text { Sim } \\
\text { Não }\end{array}$ & $\begin{array}{l}7(8,2) \\
9(10,6)\end{array}$ & $\begin{array}{l}48(56,5) \\
21(24,7)\end{array}$ & $\begin{array}{l}55(64,7) \\
30(35,3)\end{array}$ \\
\hline Aspecto Físico & $\begin{array}{l}\text { Sim } \\
\text { Não }\end{array}$ & $\begin{array}{l}14(16,5) \\
12(14,1)\end{array}$ & $\begin{array}{l}41(48,2) \\
18(21,2)\end{array}$ & $\begin{array}{l}55(64,7) \\
30(35,3)\end{array}$ \\
\hline Dor & $\begin{array}{l}\text { Sim } \\
\text { Não }\end{array}$ & $\begin{array}{l}51(60,0) \\
26(30,6)\end{array}$ & $\begin{array}{l}4(4,7) \\
4(4,7)\end{array}$ & $\begin{array}{l}55(64,7) \\
30(35,3)\end{array}$ \\
\hline Estado Geral de Saúde & $\begin{array}{l}\text { Sim } \\
\text { Não }\end{array}$ & $\begin{array}{l}21(24,7) \\
12(14,1)\end{array}$ & $\begin{array}{l}34(40,0) \\
18(21,2)\end{array}$ & $\begin{array}{l}55(64,7) \\
30(35,3)\end{array}$ \\
\hline Vitalidade & $\begin{array}{l}\text { Sim } \\
\text { Não }\end{array}$ & $\begin{array}{l}21(24,7) \\
11(12,9)\end{array}$ & $\begin{array}{l}34(40,0) \\
19(22,4)\end{array}$ & $\begin{array}{l}55(64,7) \\
30(35,3)\end{array}$ \\
\hline Aspectos Sociais & $\begin{array}{l}\text { Sim } \\
\text { Não }\end{array}$ & $\begin{array}{l}40(47,1) \\
24(28,2)\end{array}$ & $\begin{array}{c}15(17,6) \\
6(7,1)\end{array}$ & $\begin{array}{l}55(64,7) \\
30(35,3)\end{array}$ \\
\hline Aspectos Emocionais & $\begin{array}{l}\text { Sim } \\
\text { Não }\end{array}$ & $\begin{array}{c}17(20,0) \\
7(8,2)\end{array}$ & $\begin{array}{l}38(44,7) \\
23(27,1)\end{array}$ & $\begin{array}{l}55(64,7) \\
30(35,3)\end{array}$ \\
\hline Saúde Mental & $\begin{array}{l}\text { Sim } \\
\text { Não }\end{array}$ & $\begin{array}{c}7(8,2) \\
11(12,9)\end{array}$ & $\begin{array}{l}48(56,5) \\
19(22,4)\end{array}$ & $\begin{array}{l}55(64,7) \\
30(35,3)\end{array}$ \\
\hline
\end{tabular}




\section{DISCUSSÃO}

Como o objetivo deste estudo foi avaliar a QV e o nível cognitivo de idosos participantes de grupos de terceira idade existentes em um município do Rio Grande do Sul, Brasil, inicialmente é importante caracterizar o perfil dos idosos sujeitos do estudo. Observa-se maior percentual da presença feminina. A motivação feminina em participar das atividades de lazer é diferente da masculina, uma vez que a maior parte das idosas exerceu papel restrito ao ambiente doméstico. As mulheres que frequentam grupos de terceira idade, em sua maioria, não desenvolveram atividades profissionais - ao contrário, foram donas-de-casa, mães e esposas. ${ }^{15}$

Além disso, o fato de as mulheres terem participação mais ativa nos grupos de convivência pode estar relacionado ao fenômeno conhecido como a feminização da população idosa. Em números relativos, existem cerca de 100 mulheres para 82 homens idosos, fato associado à maior expectativa de vida da população feminina, o que é um fenômeno mundial. No Brasil, as mulheres vivem, em média, cerca de oito anos a mais que os homens ${ }^{16}$ e isso talvez possa explicar a maior participação feminina em grupos de convivência, evidenciando que a mulher é mais participativa e se preocupa com as questões relacionadas ao bem-estar de um modo geral, quando comparada ao homem idoso. ${ }^{16}$

Identifica-se no estudo predominância de idosos com idade entre 60 e 69 anos, o que corrobora a projeção da população idosa realizada pelo Instituto Brasileiro de Geografia e Estatística, ${ }^{17}$ segundo o qual a média da população para o ano de 2010, na faixa etária de 60 e 69 anos, será um contingente de aproximadamente 5,3 milhões de idosos. Importante salientar a participação de idosos mais jovens em grupos de convivência, cuja tendência é envelhecer e manter-se nas atividades grupais e, assim, ter um envelhecimento ativo.

Em relação à situação conjugal dos idosos estudados, $54,1 \%$ são formados por viúvos, diferentemente de outros estudos, nos quais o percentual de viuvez encontrado foi de $36,53 \%{ }^{18}$ e 49,75\%. ${ }^{16}$ A situação de viuvez, para alguns idosos, pode significar autonomia e liberdade. Quer dizer, ao mesmo tempo em que a viuvez, de modo geral, pode ser vista como sinônimo de solidão, com a morte do cônjuge, constitui uma tragédia ou uma libertação, se as idosas, quando jovens e na vida adulta, não tiveram liberdade em função das relações de gênero prevalecentes. ${ }^{19}$

Quanto ao tempo de escolaridade, observase que $52,9 \%$ dos idosos frequentaram de três a sete anos o ensino formal e $28,2 \%$, de um a três anos, apontando para um reduzido nível de escolaridade. Isto deve ser considerado quando se propõem ações educativas a este segmento populacional.

No quesito renda mensal, $88,2 \%$ dos idosos recebem de um a três salários mínimos. Este dado é importante na medida em que, segundo o Instituto Brasileiro de Geografia e Estatística, ${ }^{17}$ os idosos contribuem em 53\% dos domicílios brasileiros, com mais da metade do total da renda domiciliar, o que influencia diretamente na QV e renda da população brasileira.

$\mathrm{Na}$ amostragem geral, 62,4\% dos idosos residem acompanhados, enquanto que 37,6\% moram sós. Essas informações apresentam proporções diferentes dos números da Pesquisa Nacional por Amostragem de Domicílios (PNAD), segundo a qual mais de $85 \%$ dos idosos no Brasil residem acompanhados de parentes e apenas $11,6 \%$ vivem sozinhos ou com pessoas sem nenhum laço de parentesco. ${ }^{16}$

Ao realizar a associação entre os dados relativos aos domínios do SF $36 \mathrm{com}$ e sem declínio cognitivo e o arranjo familiar, identificouse que $62,4 \%$ dos idosos pesquisados residem acompanhados. A capacidade funcional, aspecto físico, estado geral da saúde, vitalidade, aspectos emocionais e saúde mental apresentam índices mais elevados autorreferidos em sua maioria por idosos que vivem sós e não apresentam declínio cognitivo. Em contrapartida, para os que apresentam declínio cognitivo e vivem sozinhos, todos os índices foram mais citados, com destaque para os aspectos sociais e a dor. 
No domínio relativo à atividade física, que avalia limitações para a realização de atividades de vida diária e seu impacto na vida do idoso, o estudo evidencia resultado similar a outro estudo, ${ }^{20}$ que aponta a importância da participação em atividades físicas, pois estas promovem a liberação de hormônios que induzem excitação física e mental e bom humor, mediando os batimentos cardíacos, a pressão sanguínea, a taxa de conversão de glicogênio em glicose para energia, entre outros, proporcionando, por conseguinte, benefícios físicos. Além disso, os idosos referem melhor percepção de si e de suas potencialidades, tornam-se mais capazes, ativos e interessados nas tarefas do cotidiano. ${ }^{20}$

O quesito dor encontra relevância, pois representa a medida da intensidade de dor sentida e seu efeito no trabalho habitual e nas atividades diárias. A dor, além de ser um limitador no cotidiano dos idosos e prejudicar suas atividades diárias, pode levar, em casos mais acentuados, ao isolamento social, ${ }^{21}$ até porque a dor é uma experiência sensorial e emocional ruim, decorrente de uma lesão tissular real ou potencial, cujo início pode ser súbito ou insidioso, com intensidade leve a intensa, constante ou recorrente. ${ }^{22}$

Nos domínios relativos ao estado geral de saúde e vitalidade, os dados coincidiram, o que parece lógico na medida em que quanto maior a vitalidade demonstrada pelos idosos na realização de atividades físicas, melhor será seu estado geral de saúde. Uma melhor QV está atrelada à realização de atividades físicas e à importância da criação de ações planejadas, pelos diversos segmentos sociais, na busca do envelhecimento saudável. ${ }^{9}$

Nos aspectos sociais e emocionais, os dados coincidem com os achados de um estudo realizado com idosos participantes de dança sênior, no qual o aspecto emocional foi o item que obteve maior impacto, com melhora significativa. ${ }^{20} \mathrm{O}$ referido estudo ${ }^{20}$ aponta para a importância da associação entre música, movimento e convívio social, despertando o sentimento de bem-estar e de equilíbrio emocional, reestimulando o prazer na realização dos afazeres diários.
Ainda em relação a esses domínios, as melhores médias autorreferidas são quanto aos aspectos emocionais e físicos. Nestes itens, o grupo de convivência oferece espaço de escuta e troca e a realização de atividades físicas, o que reforça e contribui para a melhoria das condições físicas, emocionais e sociais. $\mathrm{O}$ grupo de convivência mostra-se como fonte de contentamento, proporcionando ao idoso, felicidade, vontade de viver e conforto diante das limitações. ${ }^{23}$

No domínio saúde mental, dos idosos que não apresentam declínio cognitivo, 28,2\% moram acompanhados e 50,6\%, sozinhos; e $11,8 \%$ dos idosos moram acompanhados e apresentam declínio. Entre os idosos, é frequente a ocorrência de mais de uma condição crônica simultânea, o que eleva o risco de complicações, a presença de incapacidades e da instalação de quadros de dependência, que podem comprometer a QV do idoso e de seus familiares, constituindo um desafio para as políticas públicas de saúde. As modificações e os déficits causados pelo declínio cognitivo levam ao declínio funcional, com redução e perdas das habilidades para a realização das atividades da vida diária. Isto pode levar à necessidade de um cuidador que se responsabilize por ele e pelas tarefas. ${ }^{24}$

$\mathrm{Na}$ associação dos dados relativos à cognição - com e sem declínio cognitivo - e a realização de atividade física, verifica-se que $64,7 \%$ dos idosos realizam atividade física. No domínio capacidade funcional dos idosos que praticam atividade física, 56,5\% não apresentam declínio cognitivo e 8,2\% apresentam. Em relação ao aspecto físico dos idosos que realizam atividade física, 48,2\% não apresentam declínio cognitivo e 16,5\% possuem déficit cognitivo. Em relação à dor, os parâmetros se invertem, uma vez que $60 \%$ dos idosos praticantes de atividade física possuem declínio cognitivo e 4,7\% não. No aspecto estado geral da saúde e vitalidade, os percentuais são idênticos nos idosos que realizam atividade física, em que $40 \%$ não apresentam declínio cognitivo e $24,7 \%$ possuem. Nos aspectos sociais, do total de idosos que realizam atividade física, 47,1\% apresentam declínio cognitivo, e 17,6\% não. Referente aos 
aspectos emocionais, $44,7 \%$ dos praticantes de atividade física não possuem declínio cognitivo e $20 \%$ apresentam declínio. E no quesito saúde mental, $56,5 \%$ dos que realizam atividade física não apresentam declínio cognitivo, contra 8,2\% que apresentam.

Por atividade física, entende-se que é qualquer movimento corporal realizado pela musculatura esquelética voluntária e que resulta em gasto energético acima dos níveis de repouso. ${ }^{25} \mathrm{~A}$ realização regular de atividade física favorece positivamente nas variáveis fisiológicas, psicológicas e sociais. O aumento da força muscular, do fluxo sanguíneo, a melhora da flexibilidade e amplitude de movimentos, a redução do percentual de gordura, o enriquecimento dos aspectos neurais, a diminuição dos fatores predisponentes a quedas, a redução da resistência à insulina, a conservação da densidade óssea, que favorece a diminuição do risco de osteoporose, são alguns dos benefícios fisiológicos que a atividade física proporciona ao organismo. ${ }^{26}$

A melhora da autoestima, autoimagem e da estética corporal, a integração e socialização, a redução da ansiedade, de alguns casos de depressão e de aspectos cognitivos constituem benefícios que podem ser proporcionados pela realização de atividades físicas. ${ }^{26}$

Para uma boa QV, o idoso deve estar livre de doenças, estar integrado na sociedade e ter independência e autonomia - isto é, ter condições físicas e cognitivas para conduzir sua vida. Assim, dentre outros fatores, é possível afirmar que ter qualidade de vida é possuir capacidade funcional e bem-estar.

\section{CONCLUSÕES}

Neste estudo, que procurou avaliar a Qualidade de Vida e o nível de cognição de idosos que frequentam atividades grupais, identifica-se que os sujeitos são idosos jovens, viúvos, em sua maioria mulheres, com baixo nível de escolaridade e salarial. Nos parâmetros apontados pelo SF 36, constatou-se a pior média no quesito autorreferido "dor"; e as melhores médias nos itens referentes aos aspectos emocionais e aspectos físicos.

Já ao se traçar uma análise comparativa entre a cognição e a prática de atividades físicas, observa-se que os domínios capacidade funcional, aspecto físico, estado geral de saúde, vitalidade, aspectos sociais e saúde mental são referidos pela maioria dos idosos que pratica atividade física e mantém o nível cognitivo. Já os índices dor e aspectos sociais são citados por idosos que realizam atividades físicas também, mas apresentam declínio no nível de cognição.

Os objetivos propostos para o estudo foram alcançados e, a partir dele, se buscou produzir o conhecimento teórico no sentido de oferecer instrumentos e subsídios aos profissionais de saúde que trabalham com idosos, para que os mesmos, de posse do conhecimento produzido, possam entender a complexidade do envelhecimento, elaborando estratégias e programas de saúde que lhes proporcionem um envelhecimento com QV. 


\section{REFERÊNCIAS}

1. Amaral RC. Qualidade de vida do paciente transplantado renal submetido à ampliação vesical. Dissertação de Mestrado, Universidade de São Paulo, 2008. Disponível em: < www.teses.usp.br/teses/. Acesso em: 12/07/2010.

2. Lima AMM, Silva HS, Galhardoni R. Successful aging: paths for a construct and new frontiers. Interface - Comunic, Saúde, Educ., 2008,12(27):795-807.

3. Dalla Vecchia R, Ruiz T, Bocchi SCM, Corrente JE. Qualidade de vida na terceira idade: um conceito subjetivo. Rev. bras. Epidemiol [on line] 2005, 8(3): 246-52. Disponível em: <http://www.scielo.br/ pdf/rbepid/v8n3/06.pdf> Acesso em 20/10/2010.

4. Martins JJ, Schneider DG, Coelho FL, Nascimento ERP, Albuquerque GL, Erdmann AL, Gama FO. Avaliação da qualidade de vida de idosos que recebem cuidados domiciliares. Acta Paul Enferm. 2009; 22(3): 265-71. Disponível em: <http://www.scielo.br/pdf/ ape/v22n3/a05v22n3.pdf> Acesso em 12/07/2010.

5. Instituto Brasileiro de Geografia e Estatística (IBGE). Censo demográfico. 2006. Disponível em: <http://www.ibge.gov.br/> Acesso em: 07/10/10.

6. Silva GX, Costa CLA, Capistrano RDS. Nível de atividade física habitual e capacidade funcional de idosos atendidos pelo Programa Saúde da Família de Juazeiro do Norte, Ceará. EFDeportes.com - Revista Digital. Buenos Aires, 2010, Agosto, año 15, no 147 : s/p. Disponível em: <http://www.efdeportes.com/ efd147/atividade-fisica-habitual-de-idosos $>$ Acesso em: 19/09/2010.

7. Ávila R, Bottino CMC. Atualização sobre alterações cognitivas em idosos com síndrome depressiva. Rev. Bras. Psiquiatr. 2006; 28(4):316-20. Disponível em: <http://www.scielo.br/pdf/rbp/v28n4/2225.pdf>. Acesso em 20/10/2010.

8. Souza OS, Falcão JTR, Leal CC, Marino JG. Avaliação do desempenho cognitivo em idosos. Rev. Bras. Geriatr. Gerontol 2007 10(1): 29-38. Disponível em: <http://www.unati.uerj.br/tse/scielo. php.> Acesso em: 08/10/10.
9. Toscano JJO, Oliveira ACC. Qualidade de vida em idosos com distintos níveis de atividade física. Rev Bras Med Esporte 2009; 15(3):169-73. Disponível em <http://www.scielo.br/scielo.php.> Acesso em 19/10/2010.

10. Tahan J, Carvalho ACD. Reflexões de idosos participantes de grupos de promoção de saúde acerca do envelhecimento e da qualidade de vida. Saúde Soc. 2010, 19(4):878-888.

11. Izquierdo I. Memória. $2^{\text {a }}$ Ed. São Paulo: Artmed, 2011.

12. Almeida OP, Nitrini R. Demência. São Paulo: Fundação Byk, 1998.

13. Ciconelli RM. Tradução para o português e validação do questionário genérico de avaliação de qualidade de vida. 1997. 120p. Tese (doutorado em Ciências Médicas) - Escola Paulista de Medicina da Universidade Federal de São Paulo, São Paulo, 1997.

14. Ciconelli RM, Ferraz MB, Santos WS, Meinão IM, Aresma MR. Tradução para a língua portuguesa e validação do questionário genérico de avaliação de qualidade de vida SF36 (Brasil SF36). Rev. Bras. Reumatologia, Brasil, 1999; 39(3):143-150. Disponível em: http://www.scielo.br. Acesso em 20/07/2010.

15. Borini MLO, Cintra FA. Representações sociais da participação em atividade de lazer em grupos de terceira idade. Rev. Bras. Enferm. 2002; 55(5): 568-574.

16. Araujo LF, Coutinho MPL, Carvalho VAML. Representações sociais da velhice entre idosos que participam de grupos de convivência. Psicol. cienc. prof. [online]. 2005, 25(1): 118-131.

17. Instituto Brasileiro de Geografia e Estatística (IBGE). 2008. Educação melhora, mas ainda apresenta desafios. Disponível em: <http://www. ibge.gov.br/. Acesso em 09/10/10.

18. Leite MT, Moura C, Berlezi EM. Doenças sexualmente transmissíveis HIV/AIDS na opinião de idosos que participam de grupos de terceira idade. Rev. Bras. Geriatr. Gerontol.[on line] 2007: 10(3):339354. Disponível em: http://revista.unati.uerj.br/ cgi-bin/wxis.exe/iah/. Acesso em 09/10/2010. 
19. Camarano AA. Mulher idosa: suporte familiar ou agente de mudança? Estud. av. [online]. 2003,17(49):35-63.

20. Oliveira LC, Pivoto EA, Vianna PCP. Análise dos resultados de qualidade de vida em idosos praticantes de dança sênior através do SF-36. Acta Fisiatr 2009; 16(3):101-104

21. Celich KLS, Galon C. Dor crônica em idosos e sua influência nas atividades da vida diária e convivência social. Rev. Bras. Geriatr. Gerontol. 2009; 12(3): 345-359. Disponível em < http://www.crde-unati. uerj.br/img_tse/v12n3/pdf/art_3.pdf $>$. Acesso em: $19 \mathrm{I} 10 \mathrm{I} 10$.

22. NANDA. Diagnósticos de Enfermagem da NANDA: definições e classificação. Porto Alegre: Artmed, 2006.
23. Freitas CASL, Eugênio FES, Silva MJ, Lima FET, Vieira NFC, Pinheiro PNC. et al . Vivendo o envelhecer: vozes de um grupo de idosos. Rev. Enferm UFPE [On Line]. 2010; 4(1):93-100. Disponível em: <http://www.google.com.br/>. Acesso em 22/10/10.

24. Oliveira SFD, Duarte YAO, Lebrão ML, Laurenti R. Demanda referida e auxílio recebido por idosos com declínio cognitivo no Município de São Paulo. Saúde e Sociedade, 2007; 16(1):81-89.

25. Mazo GZ, Lopes MA, Benedetti TB. Atividade física e o idoso: concepção gerontológica. Sulina; Porto Alegre/RS, 2001.

26. Rolim FS, Forti VAM. Envelhecimento e atividade física: auxiliando na melhoria e manutenção da qualidade de vida. In: Diogo MJE, Neir AL, Cachioni M. (Orgs.) Saúde e qualidade de vida na velhice. Alínea; Campinas/SP, 2009. 